\title{
An Asymptotic F Test for Uncorrelatedness in the Presence of Time Series Dependence*
}

\author{
Xuexin Wang \\ School of Economics and WISE \\ Xiamen University, China
}

\author{
Yixiao Sun
}

Department of Economics

UC San Diego, USA

\begin{abstract}
We propose a simple asymptotic F-distributed Portmanteau test for zero autocorrelations in an otherwise dependent time series. By employing the orthonormal series variance estimator of the variance matrix of sample autocovariances, our test statistic follows an $\mathrm{F}$ distribution asymptotically under fixed-smoothing asymptotics. The asymptotic $\mathrm{F}$ theory accounts for the estimation error in the underlying variance estimator, which the asymptotic chi-squared theory ignores. Monte Carlo simulations reveal that the $\mathrm{F}$ approximation is much more accurate than the corresponding chi-squared approximation in finite samples. Compared with the nonstandard test proposed by Lobato (2001), the asymptotic F test is as easy to use as the chi-squared test: There is no need to obtain critical values by simulations. Further, Monte Carlo simulations indicate that Lobato's (2001) nonstandard test tends to be heavily undersized under the null and suffers from substantial power loss under the alternatives.
\end{abstract}

*Email: xuexinwang@xmu.edu.cn; yisun@ucsd.edu. Wang gratefully acknowledges the Fundamental Research Funds for the Central Universities [Grant No. 20720171043]. Sun gratefully acknowledges partial research support from the NSF [Grant No. SES-1530592]. 
Keywords: Lack of autocorrelations; Portmanteau test; Fixed-smoothing asymptotics; F distribution; Orthonormal series variance estimator

\section{INTRODUCTION}

In this study, we test the null hypothesis that a time series process is uncorrelated up to a certain order. This is a basic problem in time series analysis. There is a large body of literature on testing zero autocorrelations in a time series. These tests can be roughly categorized into time-domain autocorrelation-based and frequency-domain periodogram-based tests. The latter tests consider an infinite number of autocorrelations (see, e.g., Hong (1996) and Shao (2011), and the references therein). They are consistent in the sense that all nonzero autocorrelations can be detected. On the other hand, the former tests conventionally focus on a finite number of autocorrelations. They can obtain optimal power in some directions that practitioners are interested in.

Among the time-domain tests, as early as the 1970s, Box and Pierce (1970) and Ljung and Box (1978) proposed the so-called $Q$ test for a covariance stationary time series under an independent and identically distributed (i.i.d.) assumption. However, both economic theories and empirical studies have revealed that the i.i.d. assumption may be too restrictive. For example, the market efficiency hypothesis, rational expectation models, and optimal consumption smoothing theory all imply that the relevant process is a martingale difference (MD) sequence instead of an i.i.d. sequence. Empirical studies have further found that conditional heteroskedasticity is prevalent in financial time series, which invalidates the i.i.d. assumption. Taylor (1984), Diebold (1986), and Lobato et al. (2001) all considered testing serial uncorrelatedness under a weaker MD assumption.

In general, when a time series process is only uncorrelated, its sample autocorrelations are not necessarily asymptotically standard normal. Instead, the asymptotic covariance matrix 
of the sample autocorrelations depends on the data generation process (see, e.g., Hannan and Heyde (1972) and Romano and Thombs (1996)). In this case, Romano and Thombs (1996) and Horowitz et al. (2006) used the $Q$ test statistic with critical values obtained by block bootstrapping procedures, such as the single moving block and the blocks-of-blocks bootstraps. However, the difficulty in choosing the block size and high computational costs limit the scope of bootstrap-based tests.

Lobato, Nankervis, and Savin (2002) later proposed a modified $Q$ test based on a nonparametric estimator of the asymptotic variance matrix of the sample autocovariances. They considered the conventional increasing-smoothing asymptotics, whereas the amount of the nonparametric smoothing is assumed to grow with the sample size, but at a slower rate. Under this type of asymptotics, the proposed test statistic has a convenient asymptotic chisquared distribution under the null hypothesis. By invoking the consistency argument, the asymptotic chi-squared theory approximates the distribution of the nonparametric variance estimator by a degenerate distribution concentrated at the true variance matrix. Effectively, the theory completely ignores the estimation error in the variance estimator. Even with a delicate choice of the underlying smoothing parameter, the estimation error in the nonparametric variance estimator can still be substantial in finite samples. This explains why the chi-square-based test often exhibits a large size distortion in finite samples.

Recently, the literature has introduced alternative asymptotics to combat the aforementioned problem. Unlike the conventional increasing-smoothing asymptotics, the alternative asymptotics hold the amount of nonparametric smoothing to be fixed. Hence, they are also called the fixed-smoothing asymptotics. There is ample numerical evidence, along with theoretical results, on the higher accuracy of fixed-smoothing asymptotic approximations relative to conventional asymptotic approximations (see, e.g., Jansson (2004) and Sun, Phillips, and Jin (2008) for location models, and Sun (2014a, 2014b) for the generalized method of moments framework). In testing uncorrelatedness of time series, Lobato (2001) is among the first to 
consider alternative asymptotics. Although the asymptotic distribution of Lobato's (2001) test statistic is pivotal under the fixed-smoothing asymptotics, it is not standard, and critical values have to be tabulated by Monte Carlo simulations.

In this study, we employ the orthonormal series approach to variance estimation in the construction of a new Portmanteau test for zero autocorrelations, while allowing the time series to be otherwise serially dependent. This approach involves projecting the time series onto a sequence of orthonormal basis functions, and then taking the simple average of the outer products of the projection coefficients as the variance estimator. The number of basis functions is the smoothing parameter that underlies this orthonormal series variance estimator.

By employing the orthonormal series variance estimator of the asymptotic variance matrix of the sample autocovariances, the new Portmanteau test statistic follows an F distribution asymptotically under the fixed-smoothing asymptotics. The asymptotic F theory accounts for the estimation error in the underlying variance estimator, which the asymptotic chi-squared theory ignores. Monte Carlo simulations reveal that the $\mathrm{F}$ approximation is much more accurate than the corresponding chi-squared approximation in finite samples. Compared with the nonstandard test proposed by Lobato (2001), the asymptotic F test has the same ease of use as the chi-squared test, since the critical values of the $\mathrm{F}$ distributions are readily available in standard programming environments and software packages. Further, Monte Carlo simulations indicate that Lobato's (2001) test tends to be heavily undersized under the null and suffers from substantial power loss under the alternatives.

The remaining article is organized as follows. In section 2, we lay out the preliminaries. In section 3, we propose our new Portmanteau test and establish its asymptotic properties. In section 4, we conduct comprehensive Monte Carlo simulations, followed by an empirical application in section 5. In section 6, we conclude our study.

We use the following notation throughout the paper: $\mathbb{E}(a)$ is the expected value of the random variable $a$; " $\Rightarrow$ " indicates convergence in distribution; $i=\sqrt{-1}$ is the imaginary unit; 
and $\operatorname{Re}(\cdot)$ represents the real part of a complex number.

\section{PRELIMINARIES}

Let $\left\{y_{t}\right\}_{t \in \mathbb{Z}}$ be a real-valued covariance stationary time series with mean $\mu$. Define the autocovariance and autocorrelation functions:

$$
\begin{aligned}
& \gamma(j)=\mathbb{E}\left[\left(y_{t}-\mu\right)\left(y_{t-j}-\mu\right)\right], j \in \mathbb{Z}, \\
& \rho(j)=\frac{\gamma(j)}{\gamma(0)}, j \in \mathbb{Z} \backslash\{0\} .
\end{aligned}
$$

Given the observations $\left\{y_{t}\right\}_{t=1}^{T}$, we can estimate $\gamma(j)$ and $\rho(j)$ by their sample analogues:

$$
\begin{gathered}
\hat{\gamma}_{T}(j)=\frac{1}{T} \sum_{t=j+1}^{T}\left(y_{t}-\bar{\mu}\right)\left(y_{t-j}-\bar{\mu}\right), j \in 0, \ldots, T-1, \\
\hat{\rho}_{T}(j)=\frac{\hat{\gamma}_{T}(j)}{\hat{\gamma}_{T}(0)}, j \in 1, \ldots, T-1,
\end{gathered}
$$

where $\bar{\mu}=T^{-1} \sum_{t=1}^{T} y_{t}$ is the sample mean.

As in Box and Pierce (1970), we are interested in testing whether the first $s$ autocorrelations or autocovariances are zero. That is, we are interested in the null

$$
H_{0}^{(s)}: \gamma^{(s)}=\mathbf{0}
$$

where $\gamma^{(s)}=(\gamma(1), \ldots, \gamma(s))^{\prime}$.

The $Q$ test statistic in Box and Pierce (1970) and Ljung and Box (1978) takes the following form:

$$
\hat{Q}_{T}(s)=T \sum_{j=1}^{s} \hat{\rho}_{T}^{2}(j)
$$


or

$$
\hat{Q}_{T}(s)=T(T+2) \sum_{j=1}^{s}(T-j)^{-1} \hat{\rho}_{T}^{2}(j) .
$$

The latter is known to have better finite sample properties. Denote $\hat{\gamma}_{T}^{(s)}=\left(\hat{\gamma}_{T}(1), \ldots, \hat{\gamma}_{T}(s)\right)^{\prime}$ and $\hat{\boldsymbol{\rho}}_{T}^{(s)}=\left(\hat{\boldsymbol{\rho}}_{T}(1), \ldots, \hat{\boldsymbol{\rho}}_{T}(s)\right)^{\prime}$. Under the assumption that $y_{t} \sim$ iid, as well as other mild regularity conditions, it can be shown that, under $H_{0}^{(s)}$,

$$
\sqrt{T} \hat{\boldsymbol{\rho}}_{T}^{(s)} \Rightarrow N\left(\mathbf{0}, \boldsymbol{I}_{s}\right),
$$

and

$$
\sqrt{T} \hat{\gamma}_{T}^{(s)} \Rightarrow N\left(\mathbf{0}, \boldsymbol{I}_{s} \gamma(0)^{2}\right)
$$

where $\boldsymbol{I}_{s}$ is the identity matrix of dimension $s$. It then follows that $\hat{Q}_{T}(s) \Rightarrow \chi_{s}^{2}$, the chisquared distribution with $s$ degrees of freedom.

The chi-squared approximation is convenient. However, in the absence of further restrictions on the dependence structure of the time series apart from $H_{0}^{(s)}$, we can only expect the following:

$$
\sqrt{T} \hat{\gamma}_{T}^{(s)} \Rightarrow N(\mathbf{0}, \mathbf{\Omega})
$$

where $\boldsymbol{\Omega}$ is a general nondiagonal matrix $\Omega=\left[\omega^{(i, j)}\right]_{i, j=1}^{s}$ for

$$
\omega^{(i, j)}=\sum_{l=-\infty}^{\infty} \mathbb{E}\left[\left(y_{t}-\mu\right)\left(y_{t-i}-\mu\right)\left(y_{t+l}-\mu\right)\left(y_{t+l-j}-\mu\right)\right]
$$

The diagonal elements of $\boldsymbol{\Omega}$ can be different from $\gamma(0)^{2}$. In this case, Romano and Thombs (1996) pointed out that the $Q$ test based on the chi-squared critical values can deliver misleading results. Therefore, they proposed using computer-intensive methods, such as simple block bootstrapping and subsampling, to obtain more reliable critical values. Moreover, Horowitz et al. (2006) proposed a double blocks-of-blocks procedure with pre-whitening. However, 
these procedures are computationally intensive. They also require a user-chosen parameter, namely, the block size or the subsample size, which is difficult to pin down in finite samples.

On the other hand, Lobato et al. (2002) proposed a modified $Q$ test, which is based on the test statistic:

$$
\tilde{Q}_{T}(s)=T \hat{\gamma}_{T}^{(s) \prime} \hat{\Omega}^{-1} \hat{\gamma}_{T}^{(s)},
$$

where $\hat{\Omega}$ is a nonparametric estimator of $\Omega$. Denote $f_{t}=\left(f_{1 t}, \ldots, f_{s t}\right)^{\prime}$ with the $j$ th element given by $f_{j t}=\left(y_{t}-\bar{\mu}\right)\left(y_{t-j}-\bar{\mu}\right)$ and $\tilde{f}_{t}=\left(\tilde{f}_{1 t}, \ldots, \tilde{f}_{s t}\right)^{\prime}$ with the $j$ th element given by

$$
\tilde{f}_{j t}=\left(y_{t}-\bar{\mu}\right)\left(y_{t-j}-\bar{\mu}\right)-\hat{\gamma}_{T}(j)
$$

In general, a nonparametric estimator of $\Omega$ takes the quadratic form of

$$
\hat{\mathbf{\Omega}}=\frac{1}{T} \sum_{t=1}^{T} \sum_{j=1}^{T} w_{h}\left(\frac{t}{T}, \frac{j}{T}\right) \tilde{f}_{t} \tilde{f}_{j}^{\prime},
$$

where $w_{h}(\cdot, \cdot)$ is a weighting function and $h$ is the smoothing parameter indicating the amount of nonparametric smoothing. For example, we can take $w_{h}(t / T, j / T)=k((t-j) /(h T))$ for a kernel function $k(\cdot)$, leading to the usual kernel variance estimator. The Newey and West (1987) estimator, for instance, has this form with $k(\cdot)$ equal to the Bartlett kernel $k(x)=\max \{1-|x|, 0\}$. Notably, $\hat{\boldsymbol{\Omega}}$ is based on the demeaned moment process $\tilde{f}_{t}$. Hall (2000) showed that demeaning is essential to ensure the consistency of $\hat{\Omega}$ under the local alternatives and can help improve the testing power.

By allowing the amount of nonparametric smoothing in $\hat{\Omega}$ to grow with the sample size, but at a slower rate, Lobato et al. (2002) established that $\tilde{Q}_{T}(s)$ is asymptotically chi-squared under the null hypothesis. However, such an increasing-smoothing asymptotic approximation ignores the estimation error in the variance estimator. As a result, it can be highly inaccurate in finite samples. 
Interestingly, Lobato (2001) proposed another test statistic:

$$
\hat{L}_{T}(s)=T \hat{\gamma}_{T}^{(s) \prime}\left(\frac{1}{T^{2}} \sum_{t=1}^{T} \Upsilon_{t} \Upsilon_{t}^{\prime}\right)^{-1} \hat{\gamma}_{T}^{(s)}
$$

where

$$
\Upsilon_{t}=\sum_{j=1}^{t}\left(\begin{array}{c}
f_{1 j}-\hat{\gamma}_{T}(1) \\
\ldots \\
f_{s j}-\hat{\gamma}_{T}(s)
\end{array}\right)
$$

In this case, Lobato (2001) showed that the asymptotic distribution of $\hat{L}_{T}(s)$ is still pivotal, although it is not standard. Indeed, the alternative asymptotics here belong to the fixed-b asymptotics developed by Kiefer, Vogelsang, and Bunzel (2000). The implicit variance estimator $T^{-2} \sum_{t=1}^{T} \Upsilon_{t} \Upsilon_{t}^{\prime}$ is nothing but the Bartlett kernel estimator with the bandwidth equal to the sample size $T$ (see also Shao $(2010,2015)$ for more recent developments of this idea in the statistics literature). Although the asymptotic approximation that Lobato (2001) obtained for $\hat{L}_{T}(s)$ is more accurate than the chi-squared approximation, the asymptotic distribution is not standard, and the associated critical values have to be simulated. Further, the Monte Carlo simulation results in section 4 reveal that the test is substantially conservative.

\section{MAIN RESULTS}

In this study, we employ an alternative variance estimator - the orthonormal series variance estimator-such that

$$
w_{h}\left(\frac{t}{T}, \frac{j}{T}\right)=\frac{1}{K} \sum_{\ell=1}^{K} \Phi_{\ell}\left(\frac{t}{T}\right) \Phi_{\ell}\left(\frac{j}{T}\right),
$$

where $K$ is the smoothing parameter for this estimator and $\Phi_{\ell}(\cdot)$ satisfies the following assumption: 
Assumption 1. For $\ell=1,2, \ldots, K$, the basis functions $\Phi_{\ell}(\cdot)$ are continuously differentiable and orthonormal in $L^{2}[0,1]$ and satisfy $\int_{0}^{1} \Phi_{\ell}(x) d x=0$.

Denote

$$
\Lambda_{\ell}=\frac{1}{\sqrt{T}} \sum_{t=1}^{T} \Phi_{\ell}\left(\frac{t}{T}\right) f_{t} .
$$

Then, we have

$$
\hat{\Omega}_{\mathrm{OS}}=\frac{1}{K} \sum_{\ell=1}^{K} \Lambda_{\ell} \Lambda_{\ell}^{\prime}
$$

after a straightforward rearrangement. Notably, demeaning is not necessary here, since $\int_{0}^{1} \Phi_{\ell}(x) d x=0$. To ensure that $\hat{\Omega}$ is positive semidefinite, we assume that $K \geq s$.

In the econometrics literature, the orthonormal series variance estimator has recently been used by, for example, Phillips (2005), Müller (2007); Sun (2011, 2013, 2014a, 2014b, 2014c); Liu and Sun (2019); Lazarus, Lewis, Stock, and Watson (2016, 2018); and Martínez-Iriarte, Sun, and Wang (2019). This estimator originates from the multiple-window estimator with window function $\Phi_{\ell}(t / T)$ developed by Thomson (1982). It also belongs to the class of filterbank estimators, where $\hat{\Omega}$ is a simple average of the individual filter-bank estimators $\Lambda_{\ell} \Lambda_{\ell}^{\prime}$ (see Stoica and Moses (2005, Ch. 5)). Further, it is the sample variance of the projection coefficients (see Sun (2011) for more detailed discussions). By construction, the orthonormal series variance estimator is automatically positive semidefinite, a desirable property for practical use.

The simplest and most familiar example of this estimator is the average periodogram estimator, which involves taking a simple average of the first few periodograms. More specifically, let

$$
\Phi_{\ell}(r)= \begin{cases}\sqrt{2} \cos (\pi \ell r), & \text { if } \ell \text { is even } \\ \sqrt{2} \sin (\pi(\ell+1) r), & \text { if } \ell \text { is odd }\end{cases}
$$


Assuming that $K$ is even, we can write the resulting orthonormal series variance estimator as

$$
\hat{\Omega}_{\mathrm{OS}}=\frac{2}{K} \sum_{\ell=1}^{K / 2} \operatorname{Re}\left(A_{\ell} A_{\ell}^{*}\right),
$$

where $A_{\ell}^{*}$ denotes the transpose and complex conjugate of $A_{\ell}$ and

$$
A_{\ell}=\frac{1}{\sqrt{T}} \sum_{t=1}^{T} \exp \left(-\frac{i 2 \pi \ell t}{T}\right) f_{t} .
$$

Note that $A_{\ell}$ is the finite Fourier transform of $f_{t}$. It is exceedingly fast and convenient to compute $A_{\ell}$ in popular programming environments, such as Matlab and R. We will use this estimator in our simulation study.

With the orthonormal series variance estimator $\hat{\Omega}_{\mathrm{OS}}$, we construct the test statistic:

$$
\begin{aligned}
\tilde{Q}_{T}^{*}(s) & =\frac{K-s+1}{K s} T \hat{\gamma}_{T}^{(s) \prime} \hat{\Omega}_{\mathrm{OS}}^{-1} \hat{\gamma}_{T}^{(s)} \\
& =\frac{K-s+1}{K s} T \hat{\gamma}_{T}^{(s) \prime}\left(\frac{1}{K} \sum_{\ell=1}^{K} \Lambda_{\ell} \Lambda_{\ell}^{\prime}\right)^{-1} \hat{\gamma}_{T}^{(s)} .
\end{aligned}
$$

Note that there is a multiplicative correction term $(K-s+1) /(K s)$. When $s=1$, this term is simply equal to 1 . It becomes more important when $s$ becomes larger.

Herein, we consider the fixed-smoothing asymptotics, wherein the number of basis functions $K$ is held fixed, as the sample size increases.

\subsection{Asymptotics under the null}

To establish the fixed-smoothing asymptotics of $\tilde{Q}_{T}^{*}(s)$ under the null, we introduce the following assumption:

Assumption 2. For $r \in(0,1], T^{-1 / 2} \sum_{t=1}^{[T r]} f_{t} \Rightarrow B_{f}(r)=\Omega^{1 / 2} W_{f}(r)$, where $W_{f}(r)$ is a standard vector Brownian motion process. 
This assumption is not primitive. A more primitive condition can be found in Lobato (2001) (Assumption 2, p. 1070).

Under Assumptions 1 and 2, we have

$$
\frac{1}{\sqrt{T}} \sum_{t=1}^{T} \Phi_{\ell}\left(\frac{t}{T}\right) f_{t} \Rightarrow \int_{0}^{1} \Phi_{\ell}(r) d B_{f}(r):=\xi_{f, \ell} .
$$

Let $\eta_{f, \ell}=\Omega^{-1 / 2} \xi_{f, \ell} ;$ then, we have

$$
\eta_{f, \ell}:=\Omega^{-1 / 2} \int_{0}^{1} \Phi_{\ell}(r) d B_{f}(r)=\int_{0}^{1} \Phi_{\ell}(r) d W_{f}(r) \text { over } \ell=1, \ldots, K
$$

for each fixed $K$.

Lemma 3.1. (a) $\eta_{f, \ell} \sim \operatorname{iidN}\left(0, \boldsymbol{I}_{s}\right)$ over $\ell=1, \ldots, K$.

(b) $W_{f}(1)$ is independent of $\left\{\eta_{f, \ell}, \ell=1, \ldots, K\right\}$.

The i.i.d. property in Lemma 3.1 (a) holds because $\eta_{f, \ell}$ is normal and

$$
\operatorname{cov}\left[\eta_{f, \ell_{1}}, \eta_{f, \ell_{2}}\right]=\int_{0}^{1} \Phi_{\ell_{1}}(r) \Phi_{\ell_{2}}(r) d r=1\left\{\ell_{1}=\ell_{2}\right\}
$$

The last equality follows from the orthonormality of $\left\{\Phi_{\ell}(\cdot), \ell=1, \ldots, K\right\}$ on $L_{2}[0,1]$. Lemma 3.1 (b) holds because $W_{f}(1)$ and $\eta_{f, \ell}$ are standard normals and for $\ell=1, \ldots, K$,

$$
\begin{aligned}
\operatorname{cov}\left(\eta_{f, \ell}, W_{f}(1)\right) & =\mathbb{E}\left[\int_{0}^{1} \Phi_{\ell}(r) d W_{f}(r) W_{f}(1)^{\prime}\right] \\
& =\mathbb{E}\left[\int_{0}^{1} \Phi_{\ell}(r) d W_{f}(r) \int_{0}^{1} d W_{f}^{\prime}(v)\right]=\boldsymbol{I}_{s} \int_{0}^{1} \Phi_{\ell}(r) d r=0 .
\end{aligned}
$$


Using the continuous mapping theorem, we now have

$$
\begin{aligned}
\tilde{Q}_{T}^{*}(s) & \Rightarrow \frac{K-s+1}{K s} B_{f}(1)^{\prime}\left[\frac{1}{K} \sum_{\ell=1}^{K} \xi_{f, \ell} \xi_{f, \ell}^{\prime}\right]^{-1} B_{f}(1) \\
& =\frac{K-s+1}{K s} \Omega^{-1 / 2} B_{f}(1)^{\prime}\left[\frac{1}{K} \sum_{\ell=1}^{K} \Omega^{-1 / 2} \xi_{f, \ell} \xi_{f, \ell}^{\prime} \Omega^{-1 / 2}\right]^{-1} \Omega^{-1 / 2} B_{f}(1) \\
& =\frac{K-s+1}{s} W_{f}(1)^{\prime}\left[\sum_{\ell=1}^{K} \eta_{f, \ell} \eta_{f, \ell}^{\prime}\right]^{-1} W_{f}(1) .
\end{aligned}
$$

Note that

$$
\sum_{\ell=1}^{K} \eta_{f, \ell} \eta_{f, \ell}^{\prime} \stackrel{d}{=} \mathbb{W}\left(\boldsymbol{I}_{s}, K\right)
$$

where $\mathbb{W}\left(\boldsymbol{I}_{s}, K\right)$ is a Wishart random variable that is independent of $W_{f}(1)$. Then, by Proposition 8.2 in Bilodeau and Brenner (1999), we have

$$
W_{f}(1)^{\prime}\left[\sum_{\ell=1}^{K} \eta_{f, \ell} \eta_{f, \ell}^{\prime}\right]^{-1} W_{f}(1) \stackrel{d}{=} F_{c}(s, K-s+1),
$$

where $F_{c}(\cdot, \cdot)$ is a canonical $F$ distribution. We summarize the result in the theorem below.

Theorem 1. Let Assumptions 1 and Gold. Under $H_{0}^{(s)}$, we have

$$
\tilde{Q}_{T}^{*}(s) \Rightarrow F(s, K-s+1)
$$

where $F(s, K-s+1)$ is the standard $F$ distribution with degrees of freedom $s$ and $K-s+1$.

This result is intriguing. First, the asymptotic F test has the same ease of use as the chi-squared test, since the critical values of $\mathrm{F}$ distributions are readily available in standard programming environments. Second, the asymptotic F distribution is directly related to $K$. This is different from the conventional chi-squared theory, wherein the smoothing parameter must grow with the sample size, but does not influence the asymptotic distribution. 
Let $F^{\alpha}(s, K-s+1)$ be the $(1-\alpha)$-quantile of the $\mathrm{F}$ distribution $F(s, K-s+1)$. Our asymptotic F test is based on the statistic $\tilde{Q}_{T}^{*}(s)$ with $F^{\alpha}(s, K-s+1)$ as the critical value.

If we follow Lobato (2001) and Lobato et al. (2002) and use the test statistic of the standard form, namely, $T \hat{\gamma}_{T}^{(s) \prime} \hat{\Omega}_{\mathrm{OS}}^{-1} \hat{\gamma}_{T}^{(s)}$, we will obtain:

$$
T \hat{\gamma}_{T}^{(s) \prime} \hat{\Omega}_{\mathrm{OS}}^{-1} \hat{\gamma}_{T}^{(s)} \Rightarrow \frac{(K s)}{(K-s+1)} \cdot F(s, K-s+1)
$$

Our asymptotic F test is equivalent to the test using $T \hat{\gamma}_{T}^{(s) \prime} \hat{\Omega}_{\mathrm{OS}}^{-1} \hat{\gamma}_{T}^{(s)}$ as the test statistic and

$$
\frac{K}{(K-s+1)} \cdot s F^{\alpha}(s, K-s+1)
$$

as the critical value. The above modified $\mathrm{F}$ critical value is larger than the corresponding chi-squared critical value for two reasons. First, $s F^{\alpha}(s, K-s+1)$ is larger than the corresponding chi-squared critical value, as the F distribution has a random denominator. Second, the extra multiplicative factor $K /(K-s+1)$ is greater than 1 . This factor is larger for a larger $s$ or a smaller $K$. In finite samples, the difference between the chi-squared critical value and the modified $\mathrm{F}$ critical value can be substantial, especially when $s$ is large and $K$ is small. On the other hand, when $K$ is large, the chi-squared critical value and the modified $\mathrm{F}$ critical value will become close to each other.

In practice, we can choose $K$ to minimize the mean square error of $\hat{\Omega}_{\mathrm{OS}}$. Phillips $(2005)$ proposed such a data-driven procedure. Although it is not necessarily best suited for hypothesis testing, Monte Carlo simulations in the next section reveal that this choice of $K$ delivers good finite sample performances for the asymptotic F test. 


\subsection{Asymptotics under local alternative}

We consider the following local alternative:

$$
H_{1 T}^{(s)}: \gamma^{(s)}=\frac{\delta}{\sqrt{T}}
$$

Under this local alternative, we assume that the following functional central limit theorem (FCLT) holds:

Assumption 3. $T^{-1 / 2} \sum_{t=1}^{[T r]} f_{t} \Rightarrow r \boldsymbol{\delta}+\Omega^{1 / 2} W_{f}(r)$.

Under $H_{1 T}^{(s)}$, we now have

$$
\begin{aligned}
\tilde{Q}_{T}^{*}(s) & \Rightarrow \frac{K-s+1}{K s}\left[\boldsymbol{\delta}+\boldsymbol{\Omega}^{1 / 2} W_{f}(1)\right]^{\prime}\left[\frac{1}{K} \sum_{\ell=1}^{K} \xi_{f, \ell} \xi_{f, \ell}^{\prime}\right]^{-1}\left[\boldsymbol{\delta}+\boldsymbol{\Omega}^{1 / 2} W_{f}(1)\right] \\
& =\frac{K-s+1}{s}\left[\boldsymbol{\Omega}^{-1 / 2} \boldsymbol{\delta}+W_{f}(1)\right]^{\prime}\left[\sum_{\ell=1}^{K} \eta_{f, \ell} \eta_{f, \ell}^{\prime}\right]^{-1}\left[\boldsymbol{\Omega}^{-1 / 2} \boldsymbol{\delta}+W_{f}(1)\right] .
\end{aligned}
$$

Then, by Proposition 8.2 in Bilodeau and Brenner (1999), we obtain the following theorem:

Theorem 2. Under $H_{1 T}^{(s)}$, and Assumptions 1 and 3, we have

$$
\tilde{Q}_{T}^{*}(s) \Rightarrow F_{\lambda}(s, K-s+1),
$$

where $\lambda=\boldsymbol{\delta}^{\prime} \boldsymbol{\Omega}^{-1} \boldsymbol{\delta}$ and $F_{\lambda}(s, K-s+1)$ is the noncentral $F$ distribution with noncentrality parameter $\lambda$ and degrees of freedom $s$ and $K-s+1$.

The theorem establishes that the asymptotic F test based on $\tilde{Q}_{T}^{*}(s)$ has nontrivial testing power for the Pitman local alternative. In particular, under $H_{1 T}^{(s)}, \operatorname{Pr}\left(\tilde{Q}_{T}^{*}(s)>F_{\lambda}^{\alpha}(s, K-s+1)\right) \rightarrow$ 1 as $\lambda \rightarrow \infty$ and so the test is consistent. 


\section{MONTE CARLO EVIDENCE}

We now examine the performance of the proposed $\mathrm{F}$ test in a set of Monte Carlo experiments. The data generating processes (DGPs) used in the experiments include three MD processes and three non-MD processes. To be more precise, let $\eta_{t} \sim$ iid $N(0,1)$. The six DGPs are

M1: i.i.d. normal process: $\left\{y_{t}\right\}$ is a sequence of i.i.d $N(0,1)$ random variables.

M2: generalized autoregressive conditional heteroskedasticity or GARCH $(1,1)$ process: $y_{t}=h_{t} \eta_{t}$, where $h_{t}^{2}=0.1+0.09 y_{t-1}^{2}+0.9 h_{t-1}^{2}$.

M3: 1-dependent process: $y_{t}=\eta_{t} \eta_{t-1}$.

M4: non-MD-1 (non-martingale difference) process: $y_{t}=\eta_{t}^{2} \eta_{t-1}$.

M5: nonlinear moving average (NLMA) process: $y_{t}=\eta_{t-2} \eta_{t-1}\left(\eta_{t-2}+\eta_{t}+1\right)$.

M6: bilinear process: $y_{t}=\eta_{t}+0.5 \eta_{t-1} y_{t-2}$.

M1-M3 are MD sequences. More specifically, the i.i.d process in M1 is a basic benchmark. The GARCH process in M2 is empirically relevant in the financial literature. The 1-dependent process in M3 is considered by Romano and Thombs (1996), Lobato (2001), and Horowitz et al. (2006); for this process, $\boldsymbol{\Omega}$ is an identity matrix, except that $\omega^{(1,1)}=3$. M4-M6 are non-MD processes, but with zero autocorrelations. M5 and M6 are considered by Lobato (2001) and Horowitz et al. (2006).

To examine the size property, we repeat the experiment 10,000 times for sample sizes $T=100$ and 200. The nominal level of all tests is $5 \%$. We use values for $s$ from 1 up to 10 and 15 for $T=100$ and 200, respectively. Figures 1 and 2 report the empirical rejection probabilities of the $\tilde{Q}_{T}^{*}(s)$ test, that is, the asymptotic F test based on test statistic $\tilde{Q}_{T}^{*}(s)$. As a comparison, the empirical rejection probabilities are also reported for the $\hat{Q}_{T}(s), \tilde{Q}_{T}(s)$ and $\hat{L}_{T}(s)$ tests. The $\hat{Q}_{T}(s)$ and $\tilde{Q}_{T}(s)$ tests are based on the chi-squared approximations, and the $\hat{L}_{T}(s)$ test is based on the nonstandard distribution established in Lobato (2001). The $\tilde{Q}_{T}(s)$ test is implemented using the vector autoregression heteroskedasticity and autocorrelation 
consistent procedure described in Lobato et al. (2002), with the vector autoregression (VAR) order selected by the Bayesian information criterion. The maximum VAR order is set at $1.2 T^{1 / 3}$. This procedure differs slightly from the procedure of Den Haan and Levin (1997): it only conducts a global VAR order search, instead of searching for a unique autoregression order for each series separately. For easy references, we identify the tests with their test statistics $\tilde{Q}_{T}^{*}(s), \hat{Q}_{T}(s), \tilde{Q}_{T}(s)$, and $\hat{L}_{T}(s)$ with the understanding that different reference distributions are used for different tests.

The main features of the results are as follows:

i. For the i.i.d. process, all tests tend to work satisfactorily.

ii. For the GARCH process, the empirical rejection probabilities of the $\tilde{Q}_{T}^{*}(s)$ test are close to the nominal size of $5 \%$ for all $s$ considered. On the other hand, both the $\hat{Q}_{T}(s)$ and $\tilde{Q}_{T}(s)$ tests are heavily oversized, and their empirical rejection probabilities tend to increase for a larger $s$ when $T=100$. When $T=200$, the empirical rejection probabilities of $\tilde{Q}_{T}(s)$ come closer to the nominal size of $5 \%$, while those of the $\hat{Q}_{T}(s)$ tests are still far away from the nominal size of $5 \%$. In contrast, the $\hat{L}_{T}(s)$ test is heavily undersized, especially when $s$ is large.

iii. For the 1-dependent process, the size patterns of these tests are similar to those in the case of the GARCH process. This is expected, since the covariance matrices of the sample autocovariances of both processes are diagonal with heterogeneous diagonal elements.

iv. For the non-MD-1 process, the empirical rejection probabilities of the $\tilde{Q}_{T}^{*}(s)$ test are sufficiently controlled. However, those of the $\tilde{Q}_{T}(s)$ test worsen for a larger $s$, while those of the $\hat{Q}_{T}(s)$ test improve for a larger $s$, albeit still far from the nominal size of $5 \%$. On the other hand, the $\hat{L}_{T}(s)$ test is heavily undersized. In some cases, its empirical rejection probabilities are quite close to 0 . 
v. For the NLMA process, the size patterns of these tests are reasonably similar to those in the case of the non-MD-1 process.

vi. For the bilinear process, the $\hat{L}_{T}(s)$ test performs well when $s$ is small $(s \leq 3$ for $T=100$ and $s \leq 6$ for $T=200)$. The $\tilde{Q}_{T}^{*}(s)$ test is slightly oversized for the sample sizes considered here. On the other hand, both the $\hat{Q}_{T}(s)$ and $\tilde{Q}_{T}(s)$ tests are heavily oversized.

Overall, the $\tilde{Q}_{T}^{*}(s)$ test performs satisfactorily for all the processes considered here. The $\tilde{Q}_{T}(s)$ test is not reliable for a sample size of 100 or 200 . This is in accordance with the simulation findings in Lobato et al. (2002). Finally, the $\hat{L}_{T}(s)$ test tends to be heavily undersized, especially when $s$ is large.

For the power comparison, we consider an MA(1) process, $u_{t}=y_{t}+0.25 y_{t-1}$, where $\left\{y_{t}\right\}$ are generated according to DGPs M1-M6. Since there exists a substantial difference in the empirical rejection probabilities for different tests under the null, the critical values of these tests are adjusted such that the empirical rejection probabilities of the tests under the null are exactly $5 \%$. It should be noted that the empirical size adjustment is not practically feasible.

The simulations are carried out using 1,000 replications. Again, $s$ ranges from 1 to 10 and 15 for $T=100$ and 200, respectively. Figures 3 and 4 report the results of the experiments, where $5 \%$ is the nominal rejection probability of all tests. The main features of the results are as follows:

i. The $\tilde{Q}_{T}^{*}(s)$ test has substantial testing power, which is comparable to the $\tilde{Q}_{T}(s)$ and $\hat{Q}_{T}(s)$ tests in many cases. For example, for the non-MD experiments, the $\tilde{Q}_{T}^{*}(s)$ test is even more powerful than the $\tilde{Q}_{T}(s)$ and $\hat{Q}_{T}(s)$ tests when $s$ is small.

ii. The $\hat{L}_{T}(s)$ test, however, suffers from substantial power loss, even after the empirical size adjustment. 

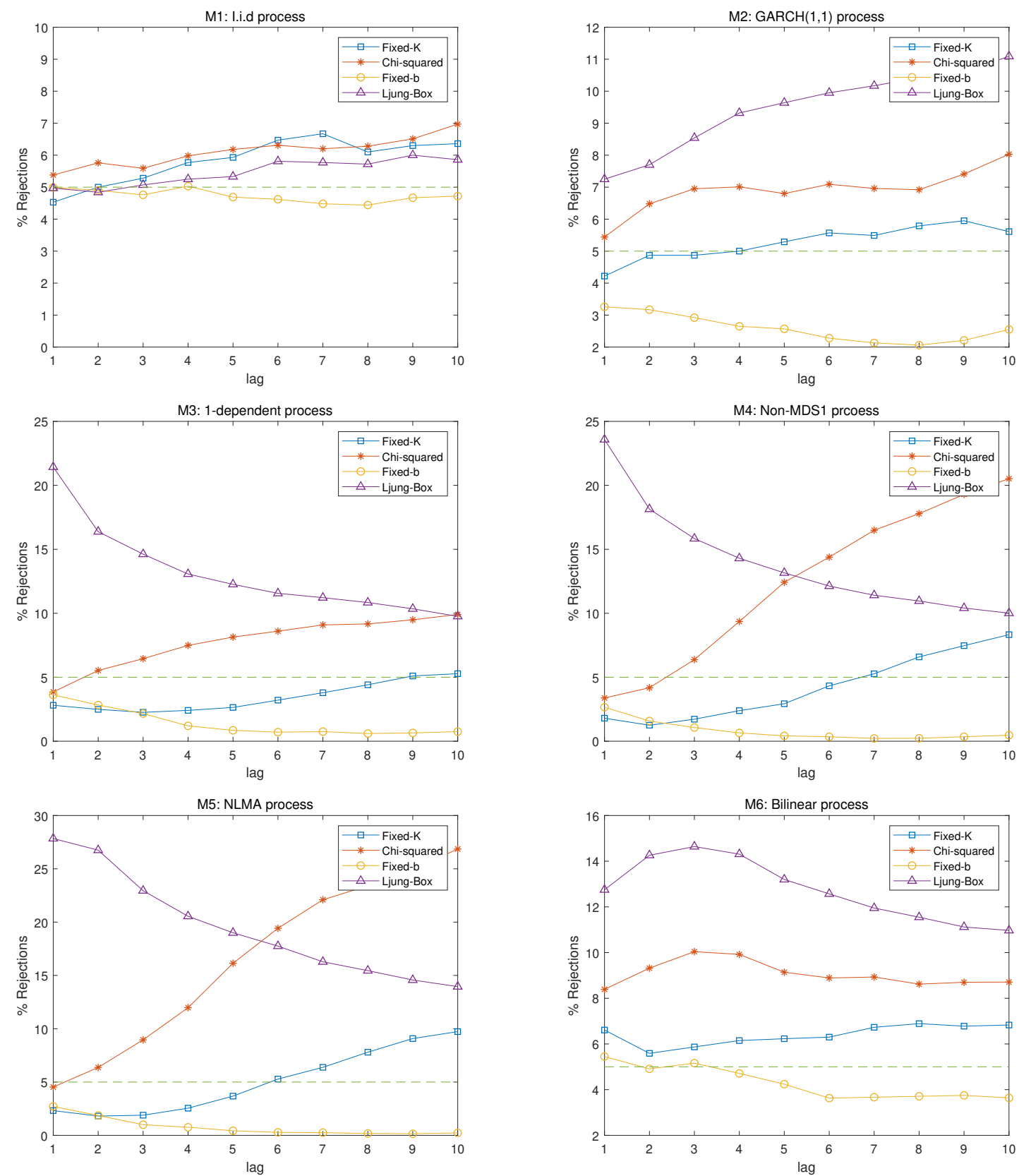

Figure 1: Percentage of rejections of Portmanteau tests in terms of the lag $s$ under the null with $T=100$. "Fixed-K" represents the asymptotic F test based on the modified statistic $\tilde{Q}_{T}^{*}(s)$ (this paper); "Fixed-b" represents the nonstandard test based on the modified statistic $\hat{L}_{T}(s)$ (Lobato, 2001); "Chi-squared" represents the Chi-squared test based on the statistic $\tilde{Q}_{T}(s)$ (Lobato et al., 2002); and "Ljung-Box" represents the Ljung-Box $Q$ test. 

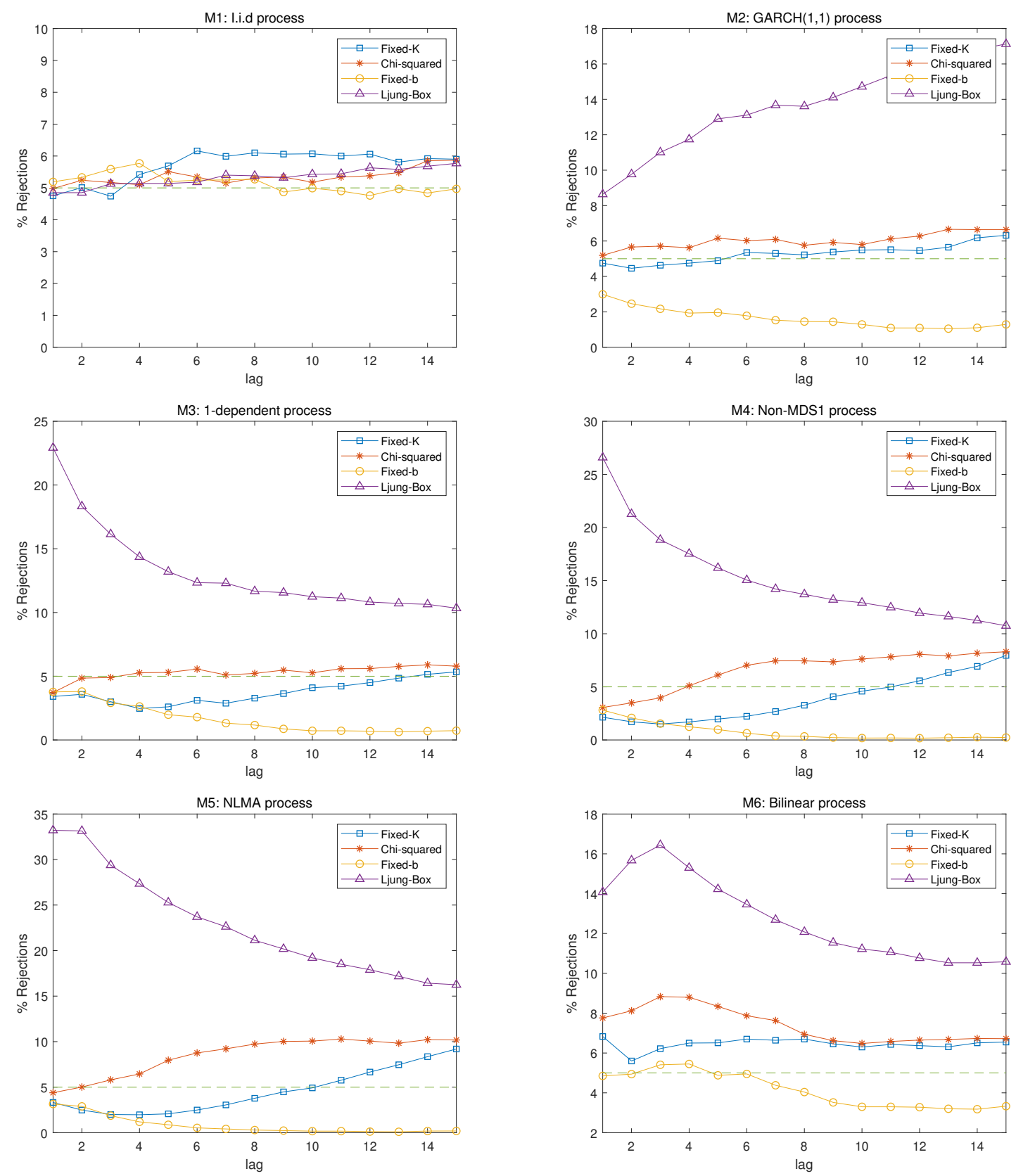

Figure 2: Percentage of rejections of Portmanteau tests in terms of the lag $s$ under the null with $T=200$. "Fixed-K" represents the asymptotic F test based on the modified statistic $\tilde{Q}_{T}^{*}(s)$ (this paper); "Fixed-b" represents the nonstandard test based on the modified statistic $\hat{L}_{T}(s)$ (Lobato, 2001); "Chi-squared" represents the Chi-squared test based on the statistic $\tilde{Q}_{T}(s)$ (Lobato et al., 2002); and "Ljung-Box" represents the Ljung-Box $Q$ test. 
In summary, the $\tilde{Q}_{T}^{*}(s)$ test has the most accurate size among all four tests, with only a minor compromise in testing power in some cases.

\section{APPLICATION}

We use the extended Nelson and Plosser (1982) economic data set for our empirical application. The data set contains annual information of 15 relevant economic series for the U.S. economy. Following Lobato (2001), we focus on two time series: the growth of employment and the returns of the S\&P 500 stock market index. The employment series covers the period from 1890 to 1988, whereas the stock index covers that from 1871 to 1988 . Hence, there are 99 and 118 observations, respectively. Figures 5 and 6 plot the employment growth and the stock index returns, respectively.

We consider the null hypotheses: $H_{0}^{(1)}, H_{0}^{(2)}$, and $H_{0}^{(3)}$. For the employment growth series, the p-values of the asymptotic F tests based on $\tilde{Q}_{T}^{*}(1), \tilde{Q}_{T}^{*}(2)$, and $\tilde{Q}_{T}^{*}(3)$ are $0.01,0.02$, and 0.01, respectively. Hence, the null hypotheses are rejected at the $5 \%$ level by our F tests. On the other hand, $\hat{L}_{T}(1)=23.9, \hat{L}_{T}(2)=38.3$, and $\hat{L}_{T}(3)=29.6$. These are all below the respective $5 \%$ critical values obtained by Lobato (2001), and thus, the null hypotheses are not rejected by Lobato's nonstandard tests.

For the S\&P 500 returns, the p-values of the asymptotic F tests based on $\tilde{Q}_{T}^{*}(1), \tilde{Q}_{T}^{*}(2)$, and $\tilde{Q}_{T}^{*}(3)$ are $0.10,0.05$, and 0.06, respectively. Roughly, the null hypotheses are not rejected at the $5 \%$ level by our F tests. For Lobato's (2001) tests, $\hat{L}_{T}(1)=27.6, \hat{L}_{T}(2)=78.9$, and $\hat{L}_{T}(3)=89.5$. Again, the null hypotheses are not rejected at the $5 \%$ level by Lobato's (2001) tests. The p-values of the chi-squared tests based on $\tilde{Q}_{T}(1), \tilde{Q}_{T}(2)$, and $\tilde{Q}_{T}(3)$ are 0.20 , 0.02 , and 0.002 , respectively. While $H_{0}^{(1)}$ cannot be rejected by the chi-squared test based on $\tilde{Q}_{T}(1), H_{0}^{(2)}$ and $H_{0}^{(3)}$ are rejected at the $5 \%$ level by the chi-squared tests based on $\tilde{Q}_{T}(2)$ and $\tilde{Q}_{T}(3)$. 

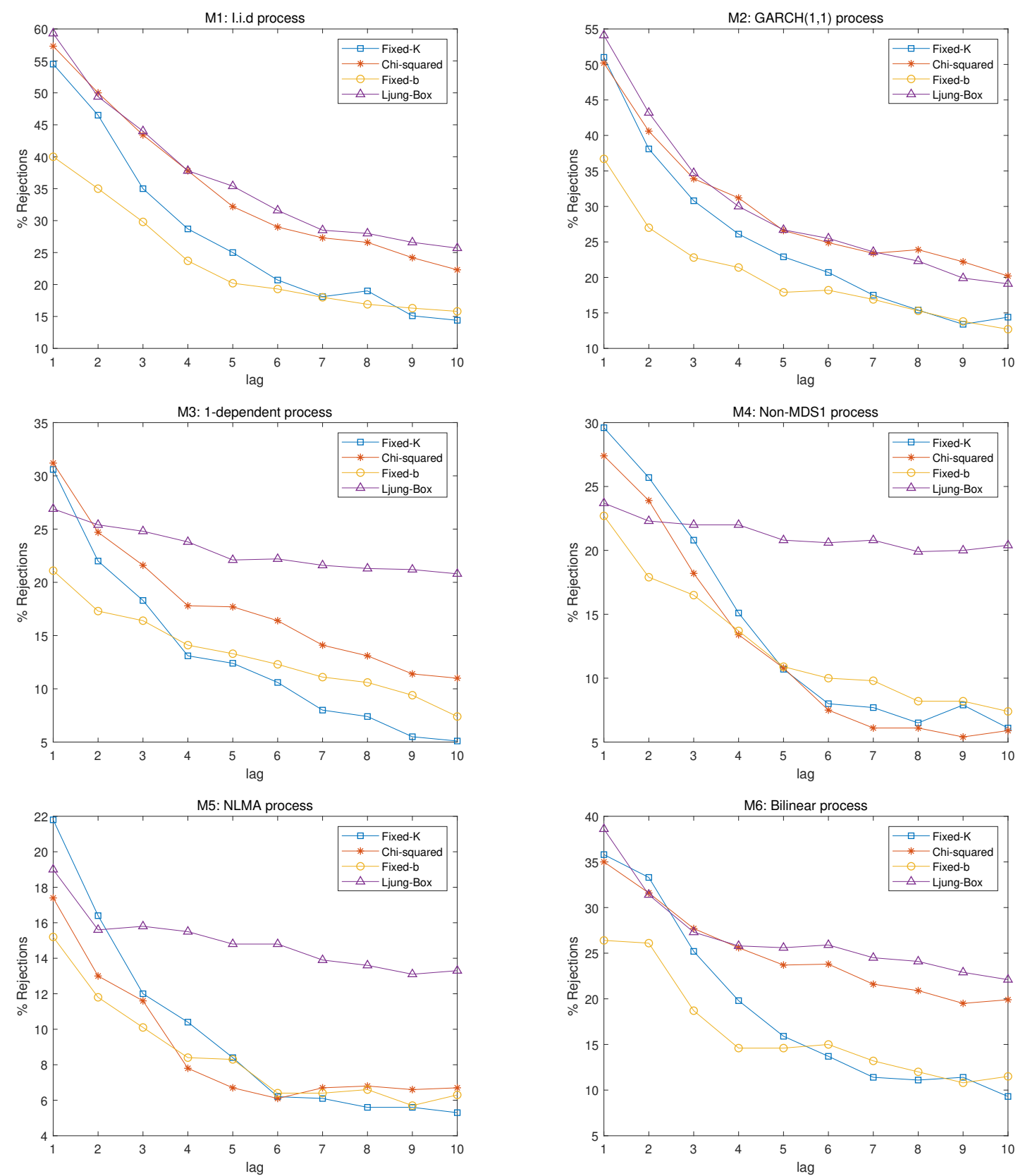

Figure 3: Sized-adjusted empirical powers of the $5 \%$ tests under an MA(1) process with $T=100$. 

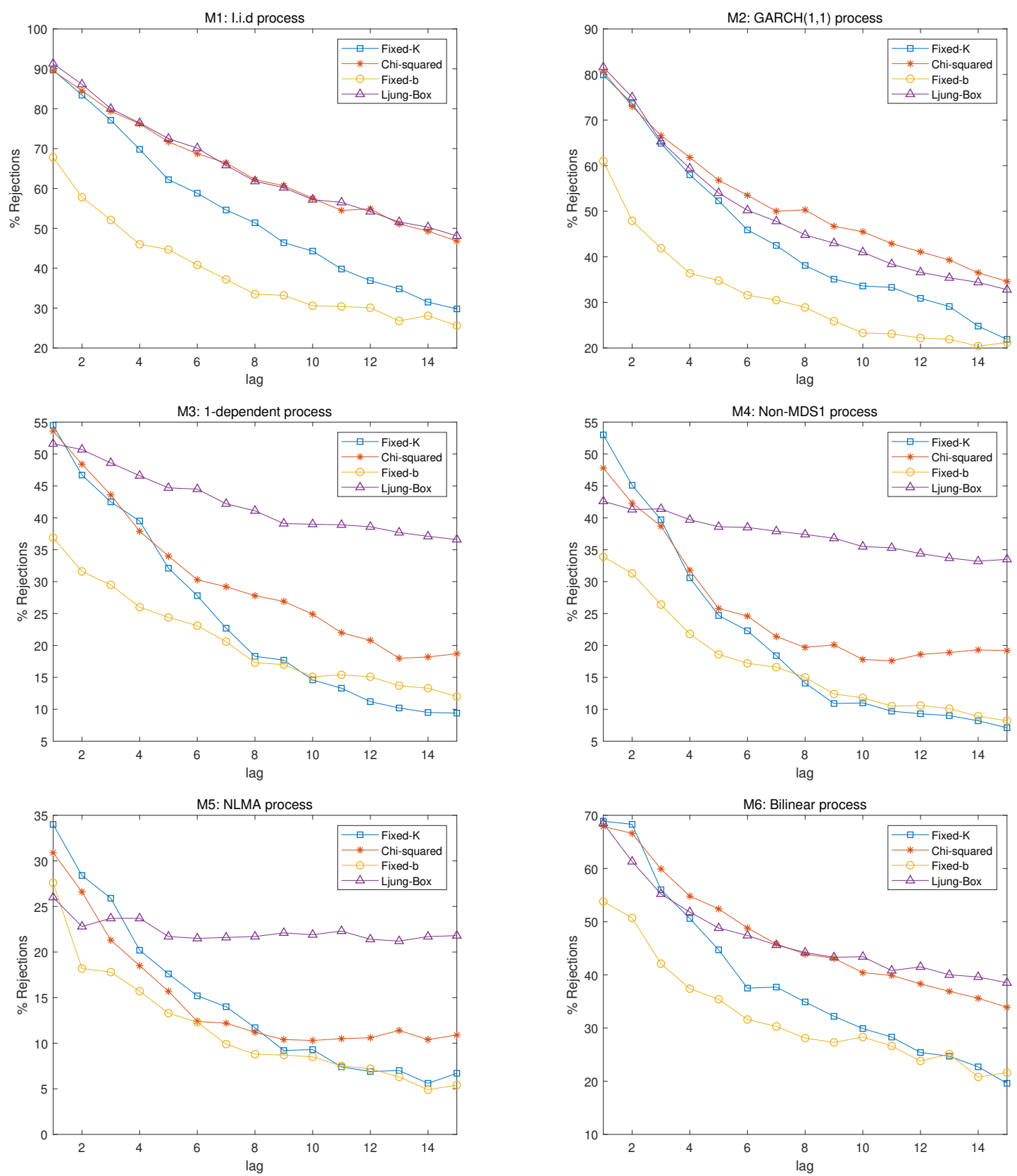

Figure 4: Sized-adjusted empirical powers of the $5 \%$ tests under an MA(1) process with $T=200$. 
Annual U.S. Employment Growth, 1891-1988

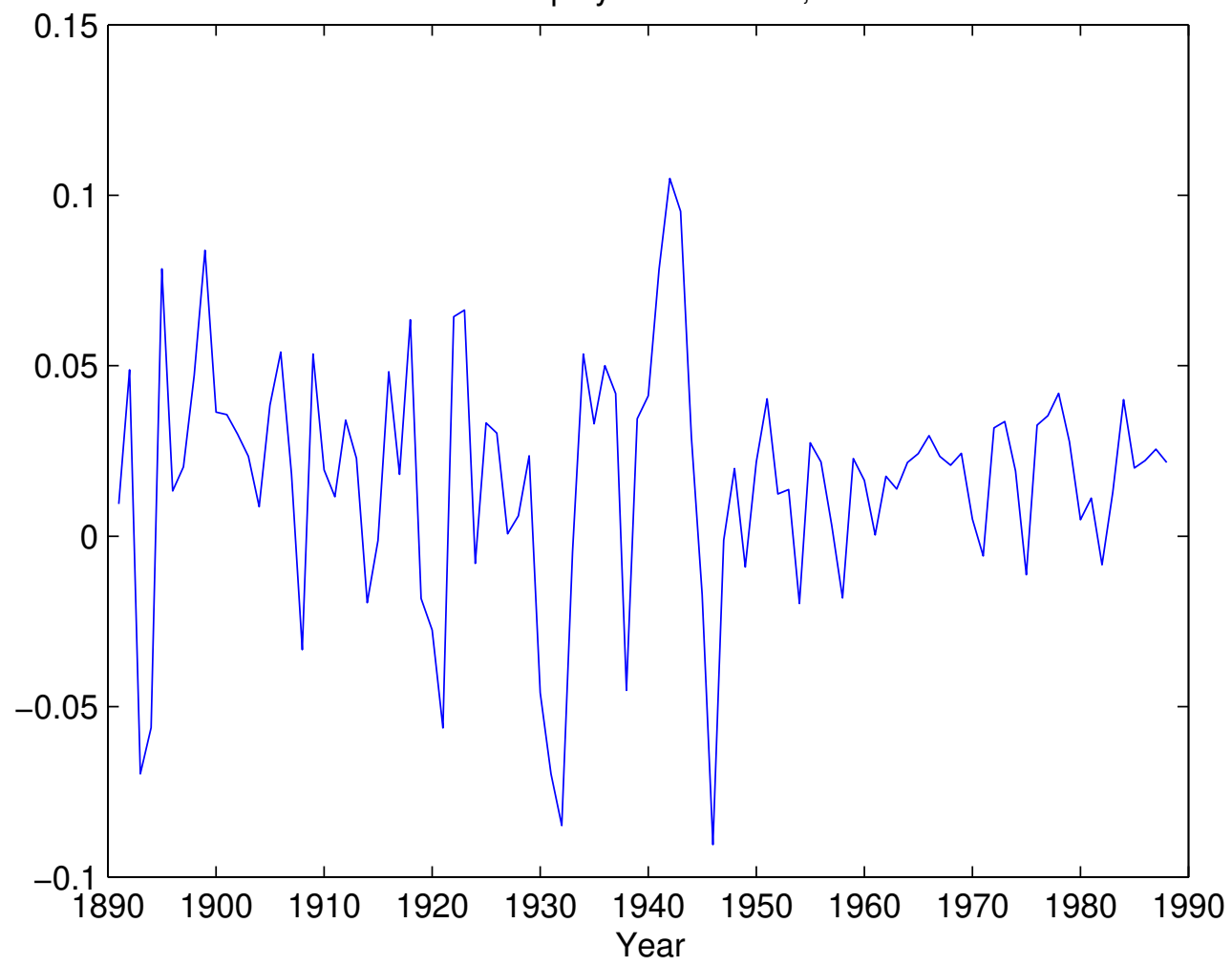

Figure 5: Time series plot of the employment growth. 


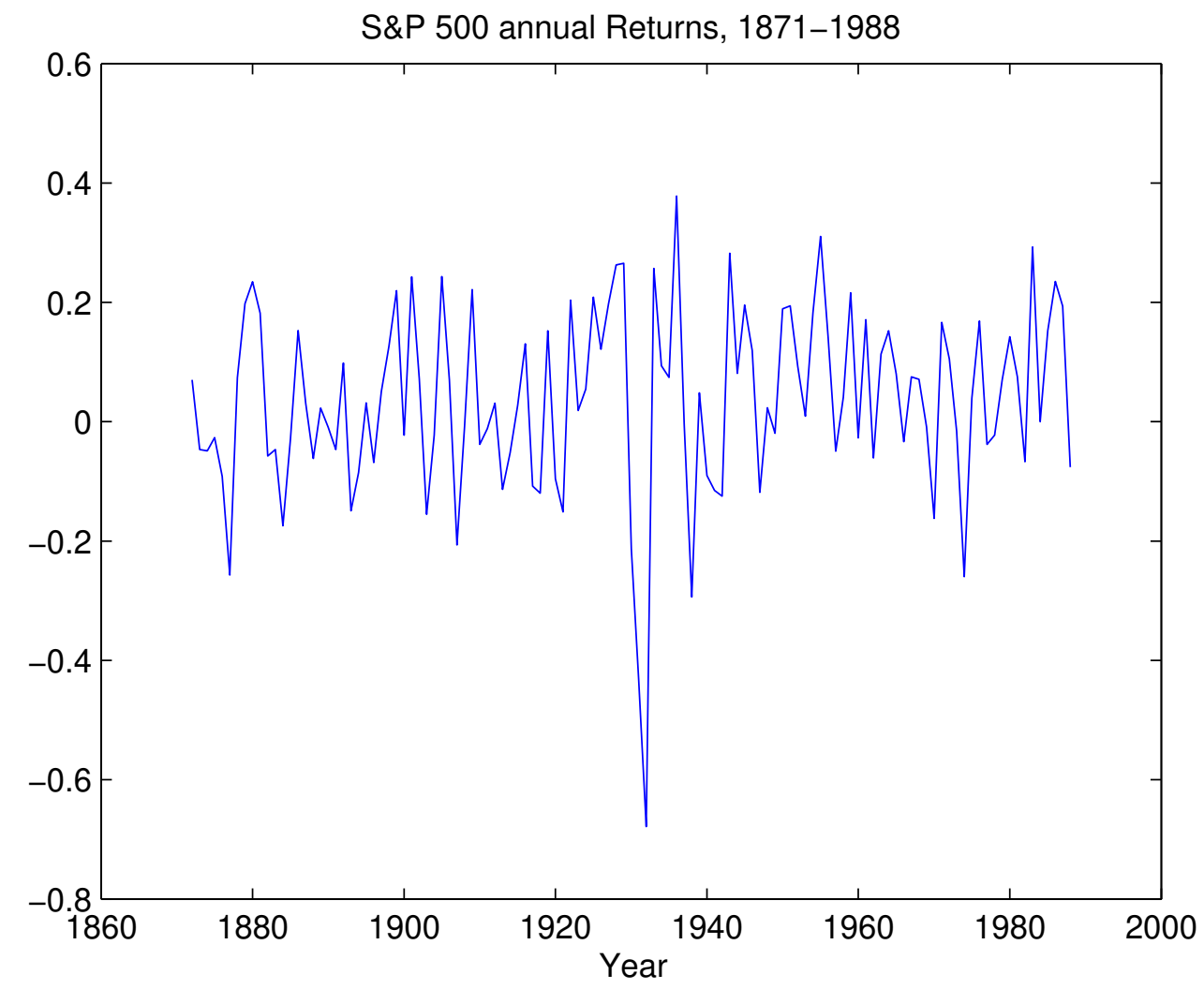

Figure 6: Time series plot of the stock index returns. 
Our results show that different tests may lead to different conclusions in empirical applications. A more accurate test is preferred in practice, as it helps us reach more trustworthy conclusions.

\section{CONCLUSION}

We propose a new test to test zero serial autocorrelations in an otherwise dependent time series. By employing the orthonormal series variance estimator of the covariance matrix of the sample autocovariances, the new test follows an F distribution asymptotically under the fixed-smoothing asymptotics. Monte Carlo simulations show that this convenient $\mathrm{F}$ test has accurate size and highly competitive power. It would be interesting to extend this methodology to test the autocorrelations of the residuals from parametric time series models. We will pursue this nontrivial extension in future research.

\section{References}

[1] Bilodeau, M., and Brenner, D. (1999): Theory of Multivariate Statistics. Springer.

[2] Box, G. E. P. and Pierce, D. A. (1970): "Distribution of the residual autocorrelations in autoregressive integrated moving average time series models." Journal of American Statistical Association 65, 1509-26.

[3] Diebold, F. X. (1986): "Testing for serial correlation in the presence of heteroskedasticity." In Proceedings of the American Statistical Association, Business and Economics Statistics Section, 323-328. 
[4] Den Haan, W. J. and Levin, A. (1997): “A practitioner's guide to robust covariance matrix estimation." Handbook of statistics 15, G.S. Maddala and C.R. Rao, eds., Elsevier, Amsterdam, 291-341.

[5] Hall, A. R. (2000): "Covariance matrix estimation and the power of the over-identifying restrictions test." Econometrica 50, 1517-1527.

[6] Hannan, E. J., and Heyde, C. C. (1972): " On limit theorems for quadratic functions of discrete time series." Annals of Mathematical Statistics 43, 2058-2066.

[7] Hong, Y. (1996): "Consistent testing for serial correlation of unknown form." Econometrica $64,837-864$.

[8] Horowitz, J. L., Lobato, I. N., Nankervis, J. C. and Savin, N. E. (2006): "Bootstrapping the Box-Pierce Q test: a robust test of uncorrelatedness." Journal of Econometrics, 133, 841-862.

[9] Jansson, M. (2004): "On the error of rejection probability in simple autocorrelation robust tests." Econometrica 72, 937-946.

[10] Ljung, G. M. and Box, G. E. P. (1978): "On a measure of lack of fit in time series models." Biometrika 65, 297-303.

[11] Lazarus, E., Lewis, D. J., Stock, J. H. and Watson, M. W. (2016): "HAR Inference: Kernel Choice, Size Distortions, and Power Losses." Working paper, Department of Economics, Harvard University.

[12] Lazarus, E., Lewis, D. J., Stock, J. H. and Watson, M. W. (2018): "HAR inference: recommendations for practice." Journal of Business and Economic Statistics 36:4, 541559. 
[13] Lobato, I. N. (2001): "Testing that a dependent process is uncorrelated." Journal of the American Statistical Association 96, 1066-1076.

[14] Lobato, I. N., Nankervis, J. C., and Savin, N. E. (2001): "Testing for autocorrelation using a modified Box-Pierce Q test." International Economic Review 42, 187-205.

[15] Lobato, I. N., Nankervis, J. C., and Savin, N. E. (2002): "Testing for zero autocorrelation in the presence of statistical dependence." Econometric Theory 18, 730-743.

[16] Müller, U. K. (2007): “A Theory of robust long-run variance estimation." Journal of Econometrics 141(2), 1331-1352.

[17] Newey, W. K. and West, K. D. (1987): "A simple, positive semidefinite, heteroskedasticity and autocorrelation consistent covariance matrix." Econometrica 55, 703-708.

[18] Phillips, P. C. B. (2005): "HAC estimation by automated regression." Econometric Theory $21,116-142$.

[19] Romano, J. P. and Thombs, L. A. (1996). "Inference for autocorrelations under weak assumptions." Journal of the American Statistical Association 91, 590-600.

[20] Shao, X. (2010): "A self-normalized approach to confidence interval construction in time series." Journal of the Royal Statistical Society B, 72, 343-366.

[21] Shao, X. (2011): "Testing for white noise under unknown dependence and its applications to diagnostic checking for time series models." Econometric Theory 27, 312-343.

[22] Shao, X. (2015): "Self-normalization for time series: a review of recent developments." Journal of the American Statistical Association 110, 1797-1817.

[23] Stoica, P. and Moses, R. (2005): Spectral Analysis of Signals. Pearson Prentice Hall. 
[24] Sun, Y. (2011): "Robust trend inference with series variance estimator and testingoptimal smoothing parameter." Journal of Econometrics 164(2), 345-366.

[25] Sun, Y. (2013): "A heteroskedasticity and autocorrelation robust F test using orthonormal series variance estimator." Econometrics Journal 16, 1-26.

[26] Sun, Y. (2014a): "Let's Fix It: fixed- $b$ asymptotics versus small- $b$ asymptotics in heteroscedasticity and autocorrelation robust inference." Journal of Econometrics 178(3), 659-677.

[27] Sun, Y. (2014b): "Fixed-smoothing asymptotics in a two-step GMM framework." Econometrica 82(6), 2327-2370.

[28] Sun, Y. (2014c): "Fixed-smoothing asymptotics and asymptotic F and t tests in the presence of strong autocorrelation." Advances in Econometrics 33, 23-63.

[29] Sun, Y., Phillips, P. C. B., and Jin, S. (2008): "Optimal bandwidth selection in heteroskedasticity-autocorrelation robust testing." Econometrica 76(1), 175-194.

[30] Taylor, S. (1984): "Estimating the variances of autocorrelations calculated from financial time series." Applied Statistics 33, 300-308.

[31] Thomson, D. J. (1982): "Spectrum estimation and harmonic analysis." IEEE Proceedings 70, 1055-1096. 